\title{
Secondary infection by anaerobic bacteria possibly ensues a battle for oxygen in SARS-Cov2 infected patients: anaerobe-targeting antibiotics (like doxycycline/Metronidazole) to supplement Azithromycin in the treatment regimen of COVID19?
}

Sandeep Chakraborty, Gautam Das ${ }^{1}$

1. Mibiome Therapeutics LLP, Mumbai.

\section{Letter}

The Covid19 pandemic [1], triggered by novel strain of a coronavirus SARS-Cov2 [2] first detected in Wuhan City, China, has spread globally like a wildfire [3], resulting in significant loss of life [4] and endangering health care providers and community health care workers [5]. Understanding and interpreting the underlying metagenome of this disease will help provide direction for the right treatment regimen.

\section{RNA-sequencing as a more sensitive and comprehensive diagnostic test:}

RNA-sequencing is a more sensitive and comprehensive test (albeit more time-consuming and expensive), providing information on a larger range of organisms (metagenomic profile) present in the patient sample in comparison to reverse transcription PCR or antibody-testing. For example in one study, Covid19 patients were tested for four bacterial species, including Mycoplasma, with negative results. However, the sequencing data clearly reveals the presence of Mycoplasma [6]. Another study of 2 patients in Wuhan [7] used the Metaphlan2 program to conclude that Capnocytophaga and Veillonella are the only bacterial species present in one patient, and found none in another, when this clearly was not the case [8].

Other RNA-sequencing data submitted in NCBI has identified several potentially pathogenic bacteria in multiple patient samples from across the globe [6,9-12]. The obligate anaerobe Prevotella had significant abundance in one patient, over-expressing immune-suppression proteins [13]. While all studies reveal Prevotella in varying abundance, other bacteria (Lautropia, Cutibacterium, Haemophilus, Pseudomonas, Sphingomonas etc.) are also found in significant quantity to attract attention to secondary infections $[6,12]$. RNA-seq also reveals extremely low viral loads in many patients, explaining the high false negatives (8 times negative before a positive) [14] and failure to detect virus using RT-PCR in severely sick patients, who were $\mathrm{CT}+$ ve $[15]$.

\section{Anaerobic bacteria hypothesis:}

Recent studies from Italy have suggested that Covid-19 does not lead to a "typical" acute respiratory distress syndrome (ARDS)' [16]. Furthermore, elevated D-dimer levels suggest hemoglobin degradation leading to coagulation $[17,18]$.

A simple hypothesis that emerges from RNA-seq data is over-representation of anaerobic bacteria in Covid19 patients, not found in BALF samples from normal patients (unpublished data), in a battle for oxygen. These bacteria express hemoglobin degrading proteins [19], heme-binding proteins sequestering heme after hemoglobin degradation [20], 'plundering' iron, and thereby sequestering oxygen [21]. Hypoxia could also result from formate, the by-product of anaerobic respiration, which inhibits mitochondrial cytochrome oxidase, causing hypoxia at the cellular level [22]. 


\section{Our proposal for anaerobe-specific antibiotics as a therapy:}

We propose the use of anaerobe-specific antibiotics, like doxycycline/Metronidazole, in the treatment regimen to supplement Azithromycin being used currently [23] since some anaerobic bacteria have resistance for Azithromycin [24] (along with anti-coagulants [25]).

\section{References}

1. Velavan TP, Meyer CG (2020) The COVID-19 epidemic. Trop Med Int Health 25: 278-280.

2. Zhu N, Zhang D, Wang W, Li X, Yang B, et al. (2020) A novel coronavirus from patients with pneumonia in China, 2019. New England Journal of Medicine .

3. Hu K, Zhao Y, Wang M, Zeng Q, Wang X, et al. (2020) Identification of a super-spreading chain of transmission associated with COVID-19. medRxiv .

4. Baud D, Qi X, Nielsen-Saines K, Musso D, Pomar L, et al. (2020) Real estimates of mortality following covid-19 infection. The Lancet Infectious Diseases .

5. Ng K, Poon BH, Puar THK, Quah JLS, Loh WJ, et al. (2020) COVID-19 and the risk to health care workers: a case report. Annals of internal medicine .

6. Chakraborty S (2020). Metagenome of SARS-Cov2 patients in Shenzhen with travel to wuhan shows a wide range of species - Lautropia, Cutibacterium, Haemophilus being most abundant - and Campylobacter explaining diarrhea. doi:10.31219/osf.io/jegwq. URL osf .io/jegwq.

7. Chen L, Liu W, Zhang Q, Xu K, Ye G, et al. (2020) RNA based mNGS approach identifies a novel human coronavirus from two individual pneumonia cases in 2019 Wuhan outbreak. Emerging Microbes \& Infections 9: 313-319.

8. Chakraborty S (2020). One of the earliest studies publishing sequencing data (Illumina) from two SARS-Cov2 patients in Wuhan failed to identify potentially pathogenic bacteria using the program Metaphlan2, when they clearly exist. doi:10.31219/osf.io/wcg4k. URL osf.io/wcg4k.

9. Chakraborty S (2020). The 2019 Wuhan outbreak could be caused by the bacteria Prevotella, which is aided by the coronavirus, possibly to adhere to epithelial cells - Prevotella is present in huge aemounts in patients from both China and Hong Kong. doi:10.31219/osf.io/usztn. URL osf .io/usztn.

10. Chakraborty S (2020). Metagenome of SARS-Cov2 from a patient in Brazil shows a wide range of bacterial species - Lautropia, Prevotella, Haemophilus - overshadowing viral reads, which does not even add up to a full genome, explaining false negatives. doi:10.31219/osf.io/2xt3w. URL osf .io/2xt3w.

11. Chakraborty S (2020). San Diego county Nanopore SARS-Cov2 sequencing data shows metagenomic Prevotella, Haemophilus parainfluenzae, a lot of unknown species and chimeric reads. doi: 10.31219/osf.io/cvbqf. URL osf . io/cvbqf.

12. Chakraborty S (2020). 'five patients at the early stage of the wuhan seafood market pneumonia virus outbreak' - bacterial load is much higher than SARS-Cov2 load, Pseudomonas is a definite problem in one patient, and Prevotella is abundant in all patients. URL osf.io/ytvuf.

13. Wu F, Zhao S, Yu B, Chen Y, Wang W, et al. (2020) Complete genome characterisation of a novel coronavirus associated with severe human respiratory disease in Wuhan. China bioRxiv 24.

14. Song L, He M, Jia X (2020) A case of SARS-CoV-2 carrier for 32 days with several times false negative nucleic acid tests. medRxiv . 
15. Jiang G, Ren X, Liu Y, Chen H, Liu W, et al. (2020) Application and optimization of RT-PCR in diagnosis of SARS-CoV-2 infection. medRxiv .

16. Gattinoni L, Coppola S, Cressoni M, Busana M, Chiumello D (2020) Covid-19 does not lead to a "typical" acute respiratory distress syndrome. American Journal of Respiratory and Critical Care Medicine .

17. Zhou F, Yu T, Du R, Fan G, Liu Y, et al. (2020) Clinical course and risk factors for mortality of adult inpatients with COVID-19 in Wuhan, China: a retrospective cohort study. The Lancet .

18. Zhang Y, Xiao M, Zhang S, Xia P, Cao W, et al. (0) Coagulopathy and antiphospholipid antibodies in patients with Covid-19. New England Journal of Medicine 0: null.

19. Guan SM, Nagata H, Shizukuishi S, Wu JZ (2006) Degradation of human hemoglobin by Prevotella intermedia. Anaerobe 12: 279-282.

20. Tong Y, Guo M (2009) Bacterial heme-transport proteins and their heme-coordination modes. Archives of biochemistry and biophysics 481: 1-15.

21. Ndongo S, Khelaifia S, Lagier JC, Raoult D (2019) From anaerobes to aerointolerant prokaryotes. Human Microbiome Journal : 100068.

22. Takahashi N, Yamada T (2000) Glucose metabolism by prevotella intermedia and prevotella nigrescens. Oral microbiology and immunology 15: 188-195.

23. Gautret P, Lagier JC, Parola P, Meddeb L, Mailhe M, et al. (2020) Hydroxychloroquine and azithromycin as a treatment of COVID-19: results of an open-label non-randomized clinical trial. International Journal of Antimicrobial Agents : 105949.

24. Arredondo A, Blanc V, Mor C, Nart J, León R (2019) Azithromycin and erythromycin susceptibility and macrolide resistance genes in Prevotella from patients with periodontal disease. Oral diseases 25 : $860-867$.

25. Tang N, Bai H, Chen X, Gong J, Li D, et al. (2020) Anticoagulant treatment is associated with decreased mortality in severe coronavirus disease 2019 patients with coagulopathy. Journal of Thrombosis and Haemostasis . 